\title{
THE LUMINOSITY FUNCTION AND EVOLUTION \\ OF COOL WHITE DWARFS
}

\author{
James Liebert
}

Steward Observatory

University of Arizona

In the review, I attempt to focus on what we have recently learned from the observations about three general topics of great theoretical interest:

1. evidence for evolution of white dwarf surface abundances,

2. the special problems of determining atmospheric parameters and abundances at the cool end of the sequence, and

3. evidence for a peaking of the white dwarf luminosity function at $M_{b o 1}=+15 \pm 1$, with possible implications for cooling theory and the history of galactic star formation.

\section{Spectroscopic Temperature/Abundance Sequences}

Figure 1 shows the parallel spectroscopic sequences of stars having hydrogen-dominated or helium-dominated atmospheric compositions. Greenstein ( 1960 and in subsequent papers)has provided the definitions and modern framework of spectral types. The non-DA sequence of course breaks into DC, metallic-line and carbon band $\left(C_{2}\right)$ stars at temperatures too cool for the DB helium lines to be seen. The middle sequence of intermediate abundance objects or "hybrids" generally contains stars with helium-dominated atmospheres and detectable trace abundances of hydrogen. The DA,F stars are included in this category since Bessell and Wickramasinghe (1979) have argued that those few objects truly deserving this classification may be hydrogen-poor. The potential $\mathrm{DC}\left(\mathrm{H}_{2}\right)$ classification recognizes the possibility of detecting $\mathrm{H}_{2}$ in cool hydrogen-rich atmospheres--see $\$ 3$.

We should not expect that each of these sequences represent the actual evolutionary stages for a star with a given atmospheric abundance. Because of several physical processes (to be reviewed next by G. Vauclair), we expect that atmospheric abundances can change as the stars cool, converting a given star from one sequence to another. I leave for him the detalled documentation of the impressive, recent theoretical work on these processes. Gravitational settling, thermal diffusion and radiation pressure may be important for hot stars, but we must especially investigate the antlcipated roles of convective mixing and accretion for the cooler stars. We should ask ourselves how it can be shown whether these predicted processes really are at work in the stars. 


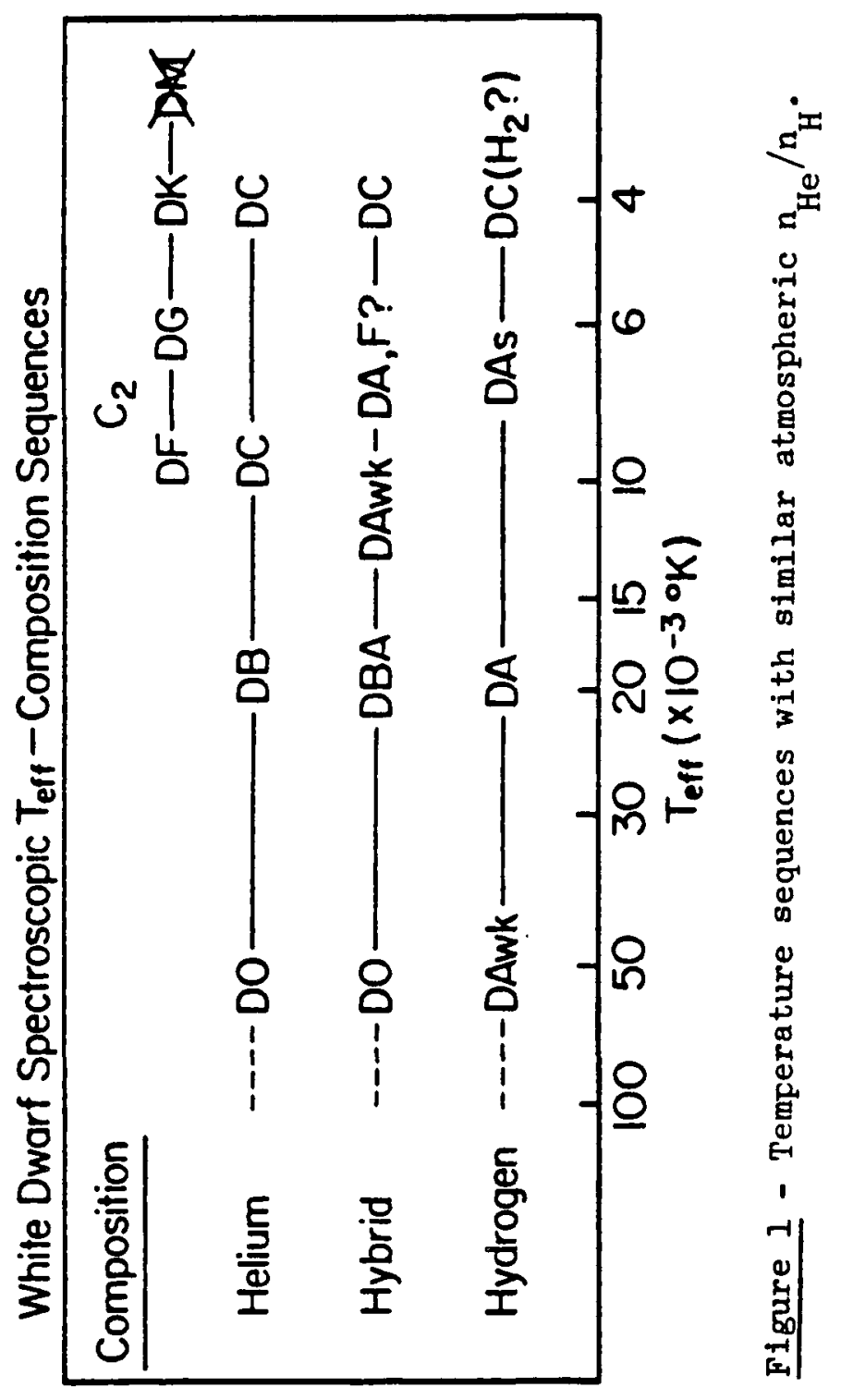




\section{Evidence for Convective Mixing}

A number of recent investigations (Koester 1976, Vauclair and Reisse 1977, D'Antona and Mazzitell1 1979a) predict convective mixing of an outer hydrogen layer into an underlying hellum envelope after the star has cooled below the DB temperature range ( $12000 \mathrm{~K}$ ). The process could quickly convert a DA atmosphere into a hydrogen-poor composition (a non-DA star). The actual surface $T_{\text {eff }}$ at which this process should occur is uncertain due to the physics in the models, but should vary from star to star depending on the values of two initial parameters $(1)$ the mass $\left(M_{H}\right)$ of the top layer of hydrogen, and (2) the total white dwarf mass. In fact, it appears that if all white dwarfs began their cooling with $\mathrm{M}_{\mathrm{H}} \succsim 10^{-7} \mathrm{M}_{0}$, the process might not occur at all and the hot DA's could all finish their cooling with hydrogen-rich atmospheres. (Note that the $10^{-} 7_{\mathrm{M}_{0}}$ of hydrogen is still orders of magnitude below the stability limit for a white dwarf.) Conversely, if all DA stars fintsh the pre-white-dwarf stage with smaller amounts of hydrogen, the calculations suggest that all stars would be converted into predominantly belium atmospheres by $T_{\text {eff }}$ $\sim 4000 \mathrm{~K}$. Hence, it is important to examine whether and to what extent convective mixing actually does take place below $212000 \mathrm{~K}$ temperatures.

For white dwarfs in the known sample estimated to be in the DB temperature range above $\sim 12000 \mathrm{~K}$, the ratio of DA to DB stars is around 3:1 (SIon and Liebert 1977). If all hotter stars are included (DAwk, DO types), the ratio is closer to $4: 1$. The question we need to address is: Does this lopsided ratio survive among the cooler stars? Recent atmospheric analyses, and better observations--particularly of the red spectral region--make possible a meaningful answer, but one must proceed with caution since many poorly-observed stars may have been misclassified. The analyses of two very cool DA stars BPM4729 (Wickramasinghe and Bessell 1979) and G128-7 (Wehrse and Liebert 1979a) show that hydrogen atmospheres can survive to temperatures as low as $\sim 5500 \mathrm{~K}$. BPM4729 is so near the low temperature limit for detection of Balmer lines that very careful observations were required; the star was originally classified DC. However, Wehrse and $I$ argued that the known stars having $5500 \approx \mathrm{T}$ eff $\delta 8000 \mathrm{~K}$ within 10 pc of the sun represent a well enough observed sample to make a meaningful statistical test of the hypothesis that a $3: 1 \mathrm{DA} / \mathrm{non}-\mathrm{DA}$ ratio survives at cooler temperatures. While the sample is small, the nearby stars have predominantly helium atmospheres by 5 to 3 . We tested against the hypothesis that the nearby stars represented a random sample from a true distribution having three hydrogen stars for every helium case; the test fails to support the hypothesis at the $97 \%$ confidence level, despite a deliberately conservative framework used in applying the test. Ed Sion will present additional evidence indicating that a lower ratio of hydrogen-to-helium atmospheres occurs among the cooler white dwarfs. The most straightforward interpretation of these results is that convective mixing really takes place, in some of the stars. This interpretation neglects any evolution in the helium-to-hydrogen ratio of the hotter sample, and does not consider accretion, though it is difficult to understand how the latter could explain a decrease in the ratio with temperature. 


\section{The Puzzle of Carbon in Non-DA Atmospheres}

The outer helium convection zones extend deeper and exist over a wider range of effective temperatures than do hydrogen convection zones. Thus, several recent theoretical investigations have focussed on the possibility that some cool helium atmospheres might show elements dredged up from deeper layers. It was initially thought that the mixed material might include ( 1 ) heavier elements which had originally sunk from the surface due to diffusion processes, and (2) the carbon, oxygen or heavier elements originally in the white dwarf core below the helium-rich envelope. However, Michaud and Fontaine (1979) and Vauclair, Vauclair and Greenstein (1979) have recently shown that the deepening convective envelope will never catch up with the diffusing material over an evolutionary time scale. Thus, the existence of detectable metals in some white dwarf atmospheres is not to be interpreted as evidence for deep dredging, but rather for accretion processes (Alcock and Illarionov 1979). A difficulty with this "consensus" view is discussed in the next section. However, it is clear that some of the highest metal abundances are observed in stars hot enough to show helium lines (e.g. GD40), which should have only shallow convection zones.

On the other hand, carbon is observed in the atmospheres of some cool stars. About one of every six known non-DA stars in the 6,000$10,000 \mathrm{~K}$ temperature range shows $\mathrm{C}_{2}(\lambda$ 4670) bands (Sion, Fragola and o'Donnell 1979). Since carbon lines have not been rellably identified in hotter helium-atmosphere stars, it has been tempting to propose that the helium envelope in the $\lambda 4670$ cases has reached a carbon-rich core. D'Antona and Mazzitelli (1979ab) argue that pre-white-dwarf mass loss should not leave a helium envelope thin enough for the convection to penetrate all the way through; however, Vauclair and Fontaine's (1979) calculations indicate that, if the helium layer were small enough, the $\mu$-discontinuity would be penetrated and carbon should be mixed upwards. Even so, they predict that the mixing should normally lead to an almost pure carbon surface composition: The known $\lambda 4670$ stars have previously been shown (Bues 1973, Grenfell i974) to fit helium-rich atmospheric models with only modest carbon enrichments $\left(\mathrm{n}_{\mathrm{C}} / \mathrm{n}_{\mathrm{He}} \simeq 10^{-3}\right.$, according to Bues 1979).

There are two difficulties in trying to test the carbon-dredging hypothesis with the available evidence:

1. it is not clear that hotter helium-atmosphere stars with $\mathrm{c} / \mathrm{He}$ abundance ratios similar to the $\lambda 4670$ stars would show readily-detectable features; and

2. while the recognized $\lambda 4670$ white dwarfs have been fit with low $\mathrm{C} / \mathrm{He}$ ratios, the expected energy distributions and spectra for any stars having much larger $\mathrm{C} / \mathrm{He}$ ratios are not well documented.

Thus, neither the empirical temperature range nor the carbon abundance range for white dwarfs is well established. Early DB atmospheric analyses by Strittmatter and Wickramasinghe (1971) and Bues (1970) suggested that atomic carbon lines would be at best marginally-detectable for $\mathrm{C} / \mathrm{He} \sim 10^{-3}$ in hotter stars; recent $\mathrm{DB}$ model calculations may clarify the fredictions. So far, the new optical spectrophotometry and 
ultraviolet spectra have not yielded any firm detections of carbon ion transitions in hot white dwarfs. Thus, we do not yet know whether carbon enrichment is a phenomenon restricted to cooler stars. Likewise, Bues' model calculations for $\lambda 4670$ temperatures indicate that the $\mathrm{C}_{2}$ band strengths may peak for increased $\mathrm{C} /$ He ratios, but that the band envelopes may blend increasingly with the carbon-dominated continuum for $\mathrm{C}>\mathrm{He}$. Is it possible, then, that white dwarfs with carbondominated atmospheres may make up part of the sample classified " $D C$ "? Unfortunately, uncertainties with the line, continuum and band opacities for carbon-dominated compositions complicate the relevant calculations; we do not yet know how DC-like the spectra and colors might be.

It is clear, therefore, that we do not understand the origin and evolution of the $\operatorname{cool} \lambda 4670$ white dwarfs. Furthermore, it is not even clear that these $C_{2}$ stars represent a higher space velocity sample compared with other white dwarfs, as most authors have previously assumed. Several examples with high space velocities have been discovered; however. Humphreys et al. (1979) have noted that most of the known $\mathrm{C}_{2}$ stars with reliable trigonometric parallaxes apparently have "old disk" space motions, as do most white dwarfs.

\section{Accretion and the Cooler Stars}

The existence of large numbers of cool degenerates whose atmospheres are free of both hydrogen and metals to very low limits poses another serious theoretical question: How are these pristine atmospheres protected from the accretion of interstellar hydrogen and heavy elements? At various times it has been argued that (1) an ionizing ultraviolet radiation flux, (2) a coronal wind driven by strong envelope acoustic fluxes, or (3) an electrostatic field in the envelope could prevent pollution of the pure helium atmospheres of the hot $D B$ stari. For the cooler hellum white dwarfs, it is much more difficult to justify these arguments, since

1. there is relatively little ultraviolet lonizing flux, especially for those hydrogen-poor cases which show strong optical line-blanketing (e.g. vMa2, G165-7);

2. the envelope acoustic fluxes are much lower for cooler helium stars than for hotter ones; and

3. the cooler hellum atmospheres are extremely deficient in free electrons and 1ons, since they are so metal-poor. Furthermore, if accretion is an occasional process requiring an encounter with an interstellar cloud (Truran et al. 1977, Wesemael 1979), the older white dwarfs are much more likely to have undergone the experience. At least the cooler hellum stars do offer deeper convective envelopes in which to dilute the pollutants, as long as the effective mean accretion rate is far below that predicted by the Bondi-Hoyle formula.

How should we in fact look for evidence that accretion has occurred in any of these stars? Of course, if accretion is a continual event or if the amount of accumulated material is large, the star would accumulate a hydrogen-rich surface abundance. Assuming a rapid sinking of the metals due to diffusion processes, the object simply 

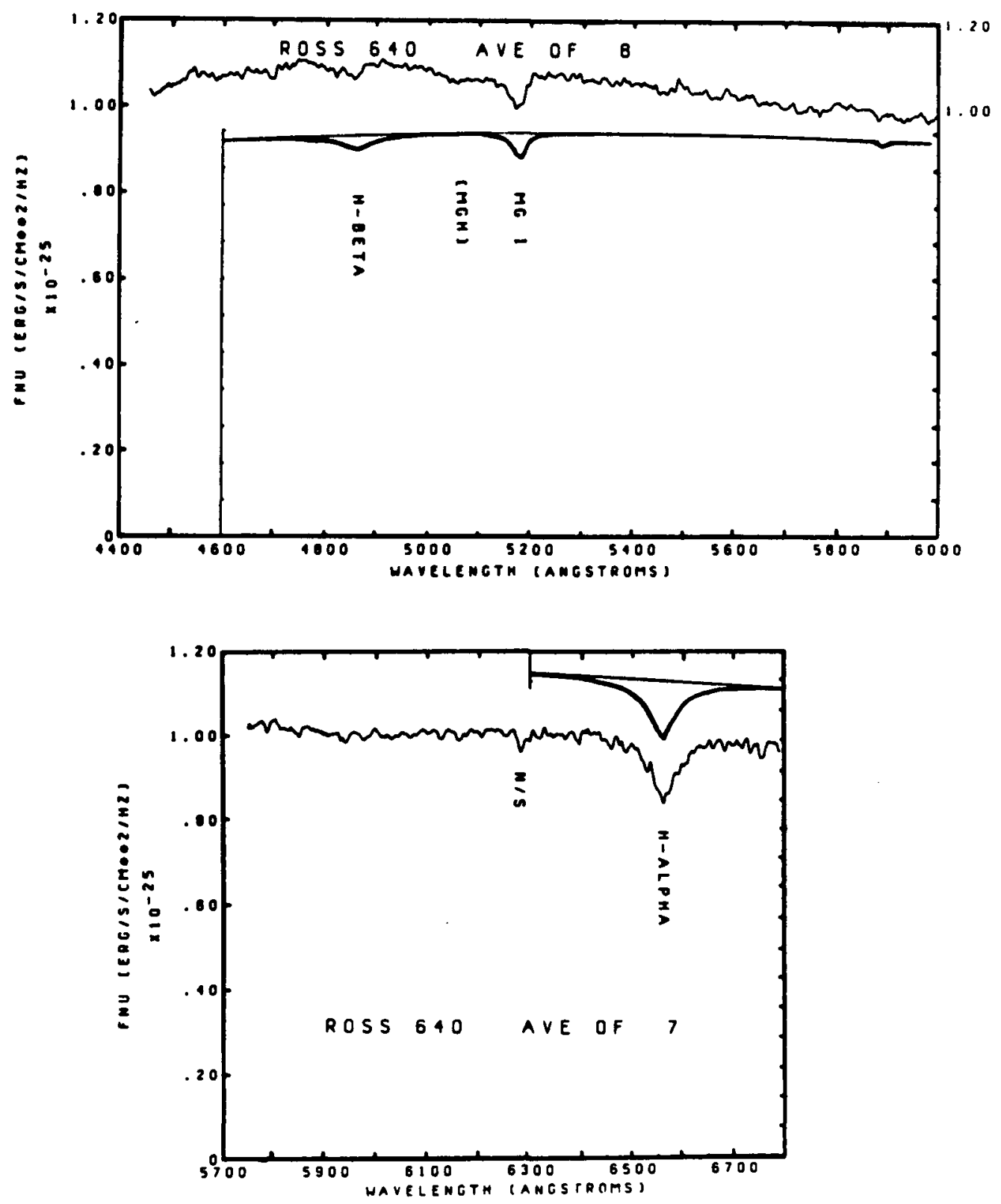

Figure 2 - Iick image dissector scanner observations of Ross 640(EGI19), compared with a model atmosphere energy distribution from Liebert(1977). 
becomes a DA star--if it wasn't one already. If the accretion is sporadic onto a cool helium-rich envelope, convective mixing into a deep layer may dilute the accreted layer so that the atmosphere remains helium-dominated, perhaps with a detectable hydrogen abundance. In this last case, we might at least for a time expect to observe something like the following:

In Figure 2 we show the red spectrum of the peculiar metallicIine star Ross 640 (from liebert 1977). The blue spectrum shows strong $\mathrm{Ca}$ II, Mg I and possible Fe I features; this red spectrum is noteworthy for the appearance of weak $H \alpha$. HB is very weak and the higher Balmer lines absent, as is expected for a small hydrogen abundance in a cool $(-8500 \mathrm{~K})$ hellum atmosphere with van der Waals rather than the Stark effect dominating the line broadening. Remarkably, it appears that the ratios of the hydrogen abundance to the abundances of the detected heavy elements are not too different from solar; in other words, the abundances of Ross 640 might be approximately reproduced by diluting some material of interstellar composition into a pure helium envelope with over 1,000 times this mass.

The recent papers by Michaud and Fontaine (1979), Vauclair, Vauclair and Greenstein (1979) and by Alcock and Illarionov (1979) conclude that the very existence of metals in such cool white dwarfs requires the supposition that accretion has recently occurred. The last authors argue that comvection or circulations due to rotation, rather than helping to maintain the abundance of metals at the surface, should actually enhance the gravitational diffusion process that would remove the accreted heavy elements from the atmosphere. Unless the process can effectively select against hydrogen in cooler stars, we would expect the hydrogen-to-metal ratios in a recently-accreting star to be solar or above, but not below solar ratios.

The problem is that, unlike the case with Ross 640 , hydrogen is usually not detected in metallic-line stars. Figure 3 demonstrates the absence of $\mathrm{H} \alpha$ in the red spectra of the strong-fined stars vMa2 and G165-7. The abundance is limited to around $10^{-4} \mathrm{n}_{\mathrm{He}}$ (Wehrse and Liebert 1979b) so that the hydrogen-to-metal ratios are distinctly below solar in these stars. An even more extreme example is presented by Greenstein's GD401, a somewhat hotter star which exhibits enormous $\mathrm{Ca}$ II lines but little else in the optical spectrum. The preliminary estimate (Greenstein 1976) was that $\mathrm{n}_{\mathrm{Ca}}$ is actually near the solar value, but $n_{H}$ must be $\leqslant 10-4 n_{\mathrm{He}}$. If one is to invoke accretion to explain the calcium abuniance in this star, the accretion process must screeen out the dominant interstellar constituent with $\geq 99.99 \%$ efficlency! Yet it is precisely these stars which tend to be relatively deficient in ultraviolet lonizing flux--due to metallic blanketing--if this must play a role in keeping out the hydrogen.

The problem of screening out the hydrogen becomes even more severe when one considers the long-term implications of assuming significant interstellar accretion onto cool helium-rich atmospheres. Alcock (1979) argues that the metal abundances are typically small in the cooler stars--and hence the metallic-line spectra are rare--because of the time dependence of the accretion process and the varying 


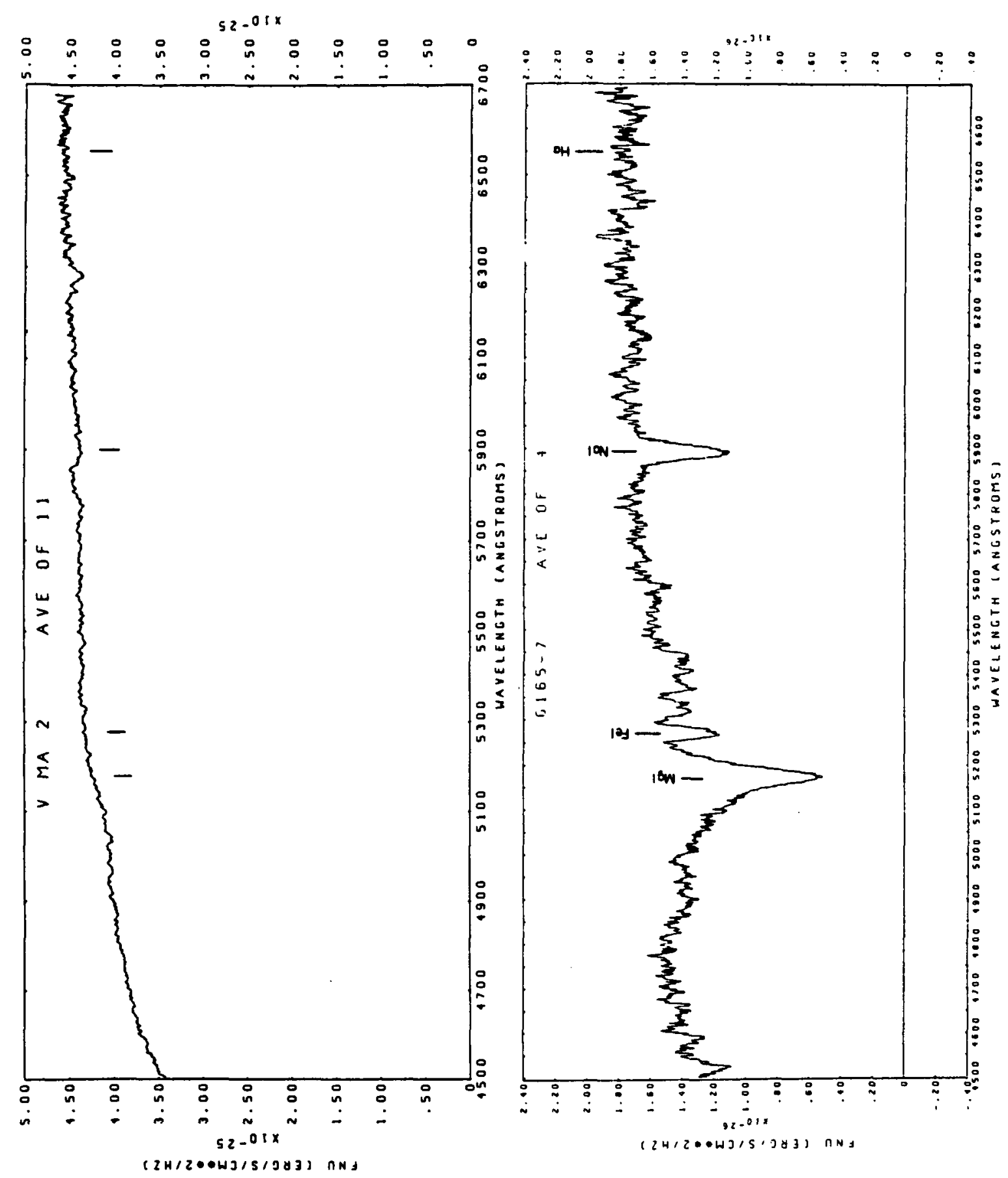

F1gure 3 - Composite Lick spectrophotometry of the red spectra of vMa2 and G165-7. 
equilibrium between the incoming mass flux and the steady gravitational diffusion. Yet, if the typical time between significant accretion events (due to cloud encounters) is only $-2 \times 10^{6}$ years, then any cool disk white dwarf should have faced a multitude of such encounters. While most of the accreted metals would have settled out of the convective envelope, all of the hydrogen would accumulate in the envelope. The implication of this analysis is that most cool helium-rich degenerates should have surface hydrogen-to-metal ratios well above the solar ratio. Thus, a highly effective screening or explusion mechanism must be found to account for the observed limits on hydrogen in large numbers of cool white dwarfs.

To summarize, since nearly all cool non-DA stars--perhaps a majorlty of all cool white dwarfs--are both extremely hydrogen- and metal-poor at their surfaces, there is not much direct empirical evidence that accretion is a generally important mechanism in determining surface abundances. If we assume that accretion is responsible for the metals detected in a few cool white dwarfs, then the scarcity or absence of hydrogen is currently a major theoretical difficulty.

\section{Abundances and Colors for the Coolest Stars}

Spectroscopic surveys by Greenstein (1979) and the Arizona group have shown that nearly all stars at the low luminosity end $-M-+15$, or $L / L_{0}-10^{-4}$--are $D C$ or nearly DC. The sole exception is the remarkable object LP701-29 (Dahn et al. 1978). Previous abundance studies, beginning with Weidemann's (1960) analysis of vMa2, showed that metal abundances and upper limits become much more stringent at the coolest $T_{\text {eff's }}$ 'secause of the reduced electron supply for the Heor $\mathrm{H}^{-}$continuum opacity. The resulting increase in atmospheric transparency leads to enormous gas pressures and densities at low optical depths for helium atmospheres. In particular, the average particle separations in helium atmospheres cooler than vMa2 must be so small that third-body interactions would affect both the continuum and line transitions and non-ideal gas effects must complicate the equation of state. Some of these problems are detailed in a recent study by $B$ blhm, Carson. Fontaine and Van Horn (1977).

It is little wonder then that the only atmospheric studies addressing abundances and line profiles below $5000 \mathrm{~K}--$ Shipman (1977) and Cottrell, Bessell and Wickramasinghe (1977)--used the assumption of hydrogen-dominated atmospheres. Shipman argued that his models were likely to be relevant to most very cool stars since the energy distributions for most of these DK-DC stars could be fit by $\mathrm{H}$ models with $\mathrm{H}^{-}$opacity. It would be quite another thing to argue that helium atmospheres could not also fit these objects, especially since the energy distributions of somewhat-hotter stars can generally be $f$ it by either $\mathrm{H}^{-}$or $\mathrm{He}^{-}$atmospheres (though with much different $\mathrm{T}_{\text {eff }}$ values).

The Cottrell et al. analysis applied only to the unique, blanketed case LP701-29. Their helium models below - $5000 \mathrm{~K}$ suggested line profiles so pressure-broadened that they would blend into the continuum. Thus, these authors argued that LP701-29 must have significant hydrogen in its atmosphere in order for the pressures to be reduced enough for any lines to be discerntble. Of course, only a few lines are 


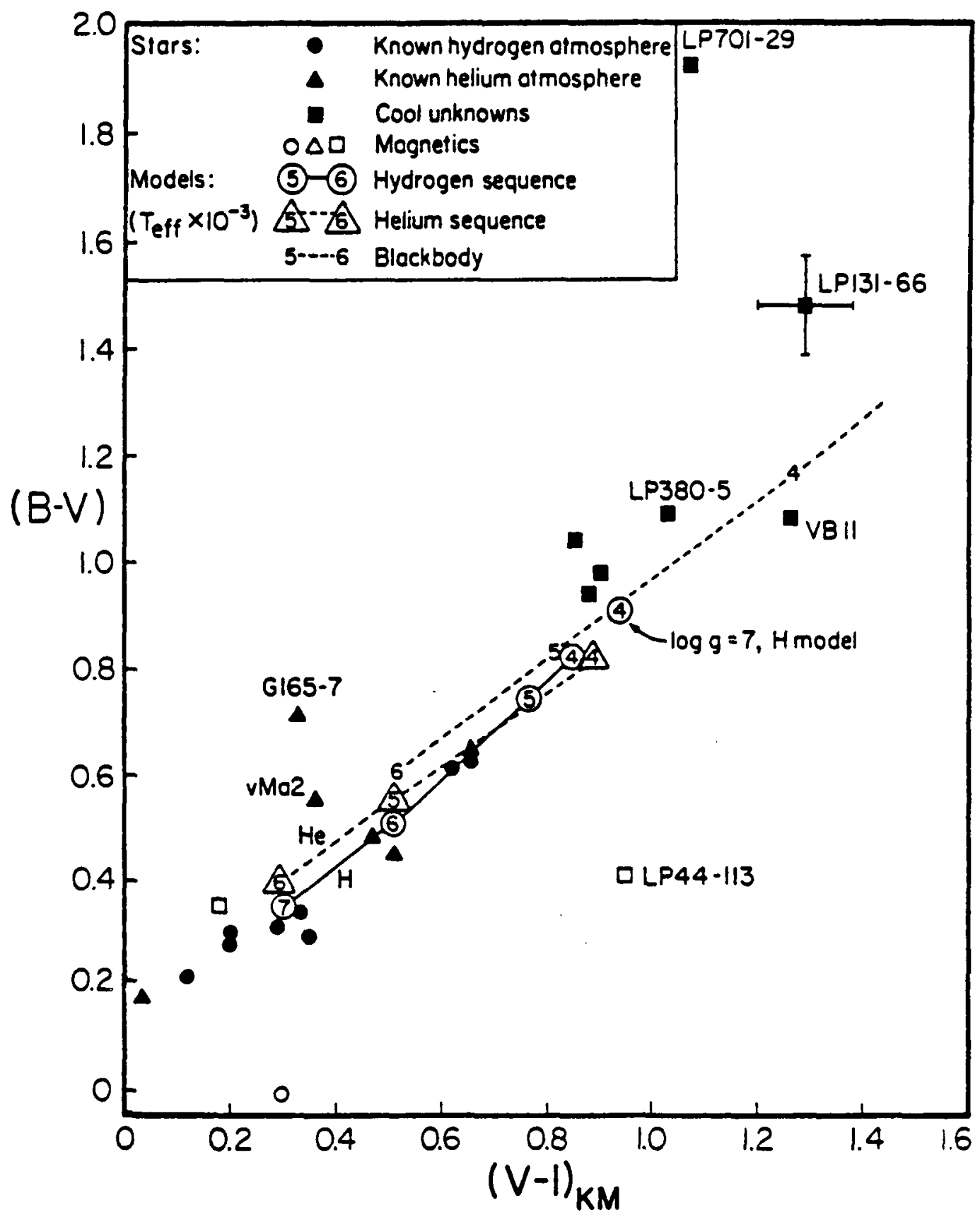

Figure 4 - Two color (BVI) diagram for cool white dwarfs, updated from Liebert et al. (1979a); colors due to C. Dahn. Models are from Mould and Iiebert (1978). Blanketed stars which are too red in ( $B-V)$ compared with the models include vMa2, G165-7, LP7O1-29, and possibly some of the coolest DK and DC stars. The energy distribution of LP44-113 (G240-72) is distorted by unknown magnetic features. 
discernible in the star's spectrum; basically, the star"s energy distribution below $4500 \AA$ is severely depressed. Furthermore, it is unclear whether the effect of pressure ionization of the metals in low temperature helium atmosphere models might permit a synthetic spectrum fit to LP701-29. Finally, there is evidence that most other cool DK-DC stars also have blue fluxes depressed by similar blanketing, though not nearly so strongly as in LP701-29.

For hydrogen atmosphere stars too cool to show Balmer lines $\left(\mathrm{T}_{\text {eff }} \leq 5500 \mathrm{~K}\right)$, most of the $\mathrm{H}$ is expected to be bound in $\mathrm{H}_{2}$ molecules anyway. The extensive series of pressure-induced $\mathrm{H}_{2}$ dipole transitions in the near-infrared spectrum offer a potential means of detecting this hydrogen. While this opacity source was recognized in exploratory papers on cool hydrogen atmospheres (e.g. Shipman 1977), it is difficult to make quantitative predictions since a large extrapolation in $T_{\text {eff }}$ from laboratory-measured (and planetary atmosphere) values is involved. However, it is reasonable to expect the opacity to be very strong in such low-temperature white dwarf atmospheres. Preliminary models and energy distributions calculated by J. Mould and myself suggested that the effect would be important for $T_{\text {eff }} \leqslant 5000 \mathrm{~K}$. Accordingly, an observational program of measuring 1-2 $\frac{f}{\mu}$ (JHK) colors was undertaken. The first results were published in Mould and Liebert (1978).

Before discussing the infrared colors, however, it is useful to show a more conventional two-color diagram for some of the coolest known white dwarfs. Figure 4 is the $\left[(B-V),(V-I)_{\mathrm{KM}}\right]$ diagram using generally $C$. Dahn's Naval Observatory measurements. Hotter white dwarf colors both with helium and hydrogen atmospheres form sequences parallel to the blackbody line in this diagram, though the temperatures fitting a given location are different for helium, hydrogen and the blackbody. Below about $5000 \mathrm{~K}$, howeyer, the model energy distributions are uncertain and the actual stars scatter much more in color. We can attribute some of this scatter to blanketing, as 1llustrated by the extreme case of LP701-29 where a deblanketing vector is needed primarily to correct $(B-V)$ and bring its location back to a position suitable for its parallax (Liebert et al. 1979a). See also the arguments in Besse1l, Wickramasinghe and Cottrell (1979).

The star LP131-66 appears to be a genuinely-unblanketed DC with both very red (B-V) and (V-I) colors; we have identified it as a possible degenerate with $\mathrm{T}_{\text {eff }}<4000 \mathrm{~K}$. A second star with apparently very red optical colors and a DC spectrum is LHS239 (LP543-32), previously discussed by Luyten, Greenstein and the Arizona group. Greenstein's multichannel colors out to one micron suggest an optical energy distribution not too different from LP131-66.

All three of these unusual stars have now been observed in the infrared, and Figure 5 is an update from the paper of Mould and Liebert. However, most of the new observations have been obtained in collaboration with $M$. Lebofsky and G. Rieke at Steward Observatory. Again, the hotter hydrogen and helium atmosphere stars follow parallel temperature sequences to - $5000 \mathrm{~K}$. Note, for example, that the colors of the very cool DA star G128-7 approximately fit the $5800 \mathrm{~K}$ 


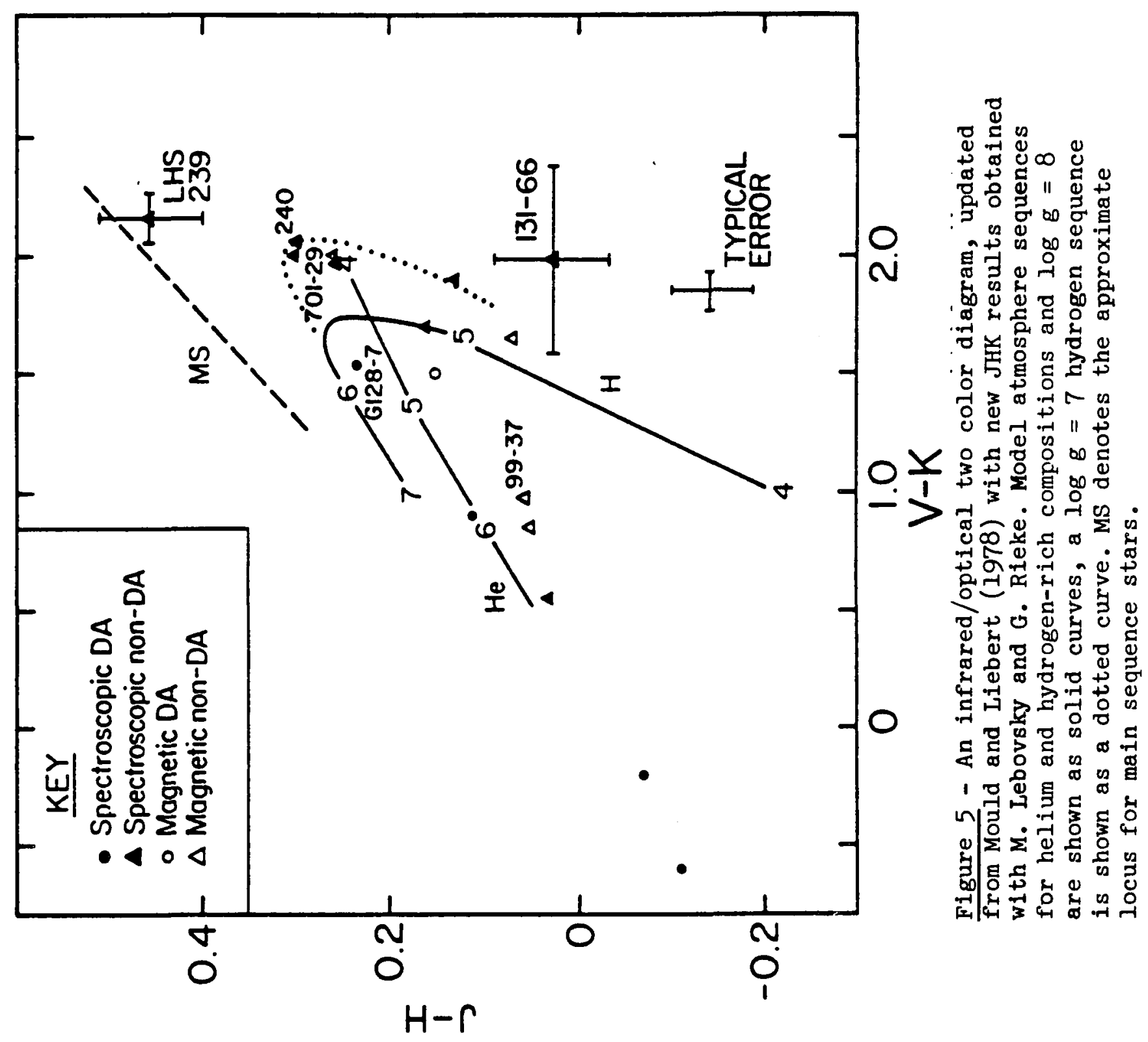


temperature derived by Rainer Wehrse and myself. Below about $5000 \mathrm{~K}$, the Mould/Liebert exploratory models using the extrapolated Iinsky opacities predict the onset of significant pressure-induced $\mathrm{H}_{2}$ dipole opacity. This causes both $(\mathrm{J}-\mathrm{H})$ and $(\mathrm{V}-\mathrm{K})$ to reverse direction at cooler temperatures due to the depression of infrared flux. Thus the hydrogen and helium sequences at lower temperatures are predicted to diverge. Unfortunately, most of the known very cool degenerates $\left(\sim 10^{-4} L_{\theta}\right)$ lie very near the junction point; they are not quite cool enough to allow separation of the hydrogen from the helium cases.

LP701-29 11ves in the clump of stars near this turning point. Its parallax indicates a slightly lower luminosity than the others, but the temperature is evidently too high to reach a conclusion from this technique as to its atmospheric composition.

Now, consider the positions of the new unblanketed stars with very red optical colors--LPI31-66 and LHS239: LHS239 clearly has quite strong infrared fluxes. LP131-66, on the other hand, was so faint at JHK that it was very difficult to measure with the Steward 2.3-m telescope and Rieke's excellent helium-cooled infrared detector. Hence, the error bars are large despite five independent measurements of this crucial object. It appears to have a discrepant ( J-H) color, in the sense predicted for the onset of the dipole opacity. Further measurements, probably on a very large telescope, are desirable in order to confirm this tentative result that LP131-66 is a very cool white dwarf with some hydrogen in its atmosphere. If the infrared result is verified, the star evidently has much more I-band (a $8000 \AA$ ) flux than is predicted by our hydrogen models (Figure 4). This would imply that the effect of the $\mathrm{H}_{2}$-dipole opacity has generally been overestimated in the models for $\log g=8$.

\section{The Luminosity Function: A Probable Deficit of Very Cool White Dwarfs}

Selection effects have greatly complicated the empirical determination of the white dwarf luminosity function at lower luminosities. Selection of hot white dwarf candidates is quite easy, using the criteria of significant proper motion and a bluish photographic color estimate. Indeed, Luyten's catalogues of white dwarfs already identify large numbers of the bluer degenerates, though Green's (1977 and in preparation) Palomar survey of bright blue objects regardless of motion has now found additional white dwarfs forming the low tangential velocity part of the true distribution. For the cooler stars, the search is stifled by (I) increasing confusion of the candidates with numerous main sequence and high velocity subdwarf stars having similar proper motion and colors, and (2) a greatly reduced search volume, since the cooler degenerates have lower luminosities. Thus the initial efforts to find lower luminosity degenerates of around solar temperature had frustratingly low yields (Greenstein 1970). Likewise, the inclusion of detailed physics in the cooling theory initially led to predictions that the stars might cool rapidly at lower luminosities anyway; then, the expected number at cooler temperatures would be far below that predicted by the Mestel relation. More recent theoretical 
calculations (Lamb and Van Horn 1975, Shaviv and Kovetz 1976) indicate, however, that the onsets of crystallization and Debye cooling in the core and convection in the envelope still leave the stars with plenty of time to spend at lumitosities at and below $10^{-4} \mathrm{~L}_{\rho^{\circ}}$ Spectroscopic survey efforts by Greenstein and by Hintzen and Strittmatter (1974) were successful in greatly increasing the known numbers of cooler degenerates. In fact when Kovetz and Shaviv (1976) and Sion and Liebert (1977) estimated the empirical luminosity distribution for a sample chosen by volume-completeness arguments, the result--as found much earlier by Weidemann (1967)--was that the numbers of white dwarfs per unit magnitude interval were still increasing at $\sim 10^{-4} \mathrm{~L}{ }_{0}$; this is consistent with the most recent theoretical predictions and the assumption of a constant birthrate. Likewise, Green (1977) noted that the number of known cool white dwarfs within - 5 pc of the sun is consistent with that expected from normalizing a theoretical cooling curve (for $L \geq 10^{-4} L_{\theta}$ ) to his empirical function for hot white dwarfs.

Unt1l very recently, however, there has been almost no observational information on white dwarfs with $\mathrm{L}<10^{-4} \mathrm{~L}_{\theta^{\circ}}$ Recent spectroscopic survey efforts, accordingly, have concentrated on the fainter Luyten Palomar survey stars having substantial motions; the efforts include our group at Arizona, Greenstein at Caltech, Hintzen and others. The Arfzona results are discussed in Liebert (1978) and Llebert, Dahn, Gresham and Strittmatter (1979b).

The Arizona program emphasized observations of all Luyten Palomar (LP) stars of faint apparent magnitude with $\mu \geq 1$ "/year in the northern two thirds of the sky $\left(\delta \geq-20^{\circ}\right)$; Greenstein had already observed all brighter Lowell survey stars with $\mu \geq 1$ ". The purpose was to detect cooler white dwarfs of very low luminosity-(VLI) defined as $+16 \leq \mathrm{M}_{\mathrm{bol}} \leq+17$ having distances $\leq 10 \mathrm{pc}$ and with tangential velocities $\geq 47 \mathrm{~km} / \mathrm{sec}\left(I^{\prime \prime} / \mathrm{yr}\right.$ at $\left.10 \mathrm{pc}\right)$. It was felt that the photographic color information was useless and even potentially misleading since unblanketed VLL degenerates would have the colors of early M stars, while any blanketed cases like LP701-29 might have extremely red colors. Hence, all large motion stars regardless of color class were observed.

The survey found new low luminosity M dwarfs, cool subdwarfs with high space velocity, and background white dwarfs with $\mathrm{M}_{\text {bol }} \leq+15$, but only one object--LP131-66--which is a possible VLL degenerate with $\mathrm{d}<10 \mathrm{pc}$.

We compared our empirical results with the predictions of (1) the Shaviv-Kovetz (1976) $0.6 \mathrm{M}$ cooling curve for a carbon-oxygen configuration, (2) normalized to Green's (1977) empirical density of non-close-binary hot white dwarfs, and (3) using initially the assumption of a constant white dwarf birthrate over the cooling range of relevance--up to 9 billion years for the VLL interval. That is, we compared the numbers of non-close-binary white dwarfs expected within our "restricted 10 parsec volume" with moderate to high tangential velocities versus our actual findings. The expected number was around 10; we found at most one object. 
There are a number of problems in interpreting these results. The foremost worry was the completeness of Luyten's catalogues for $\mu \geq l^{\prime \prime}$ and especially $16.0 \leq m_{R} \leq 18.0$, where $m_{R}$ is his machinemeasured red Palomar plate magnitude estimate. This question is discussed extensively in Liebert et al. (1979b); our conclusion, based on checks of Luyten's technique and the predicted numbers of 1 " motion stars is that Luyten (1976) should be $z 50 \%$ complete for the specific sample we are concerned with. Even so, the Arizona program was just a first attempt at constructing a quantitative framework for comparison of observations with theory. The numbers we dealt with were small enough that we would have been uncomfortable with concluding that there is a real deficiency except for other kinds of evidence strongly supporting this conclusion and the fact that the predicted numbers are so large. Greenstein (1979 et cet.) has obtained more spectra of cool proper motion stars than the rest of us put together! His recent efforts, those of Hintzen (1979) and also Wickramasinghe, Bessell and others have included spectra for over 100 faint Luyten stars, largely with motions < 1"/year. Eggen has obtained photometry for large numbers of bright Southern Hemisphere proper motion stars and published catalogues (Eggen 1979a, b) for all stars with motions $\mu \geq 1$ "/year and $0.7 " /$ year. Since the submission of the Arizona group paper, Mike Bessell, Conard Dahn, Wieslaw Wisniewsk 1 and $I$ have observed over 50 additional faint Luyten stars, most with motions < ${ }^{\prime \prime}$. The number of new VLL degenerates reported from these efforts is zero.

We can ask where are the degenerates lower in luminosity than Wolf 489 and vMa2 within several parsecs of the sun? The luminosity function would predict several such stars. These stars might be bright enough for the Lowell and early Luyten proper motion lists. of course, isolated stars at 15-16th magnitude could easily have been missed. Yet, if they were members of binaries, the chances of their being missed would be almost as low as the chances of missing the brighter companion.

Many of the known cool white dwarfs near the sun are, in fact, members of common proper motion binary systems, all with brighter companions, and most distant cases have preferentlally been discovered in this manner. By taking deep photographs of the fields of known nearby stars, Van Biesbroeck $(1944,1961)$ some $20-30$ years ago discovered faint companions which are st1ll among the lowest luminosity known $M$ dwarfs, subdwarfs and degenerates. Yet all of the luyten Palomar survey and virtually all of the faint star parallax work has been done since Van Biesbroeck's discoverles. The U. S. Naval Observatory routinely takes deep photographs of parallax star flelds to look for faint companions; they have so far found neither $M$ dwarfs as late as VB10 nor white dwarfs lower in luminosity than LP701-29. More importantly, hundreds of trigonometric parallax stars are also listed in the LP catalogue. It is important to note that Luyten routinely blinks the field of every new machine-identified star of significant proper motion in order to verify the object and to look for a motion companion. Since he generally uses the red LP plates, companions down to at least $m_{R}=19$ (or $V$ generally - 19.5) should be found in this way, and Luyten has catalogued many hundreds of common proper motion stars. 
Consider the Implications of this for the - 1300 stars within about 20 pc 1isted in the Gliese (1969, 1979) catalogue, the great majority of which were also measured by Luyten. VLL degenerate companions at least as faint as $M_{v}=+18$ should have been found; the apparent magnitude difference from a star with at least a fair luminosity estimate would identify a very low luminosity companion, either main sequence or degenerate. Now the luminosity function predicts some 250 VLL degenerates within 20 pc for the region of completed LP flelds. If we assume that these degenerates have the same frequency in separated binary systems as the dwarf $M$ 's in Gliese (1969), some 50-60 could be found in binaries. Alternatively, if we adopt the local luminosity function of Wielen (1974), the 250 VLL white dwarfs would be $-7 \%$ of the total number. If this were assumed to be representative of the luminosity function of separated binary stars as well--see evidence in Abt and Levy (1976)--then the 420 separated companions listed by Gliese should have included some 30 VLL degenerates. Note that dozens of cool white dwarf companions are listed in the Gliese catalogue, but where are the stars fainter than VB11? Could it be that proper motion pairs which must be very old would have broken up because of perturbations by third bodies? No, King (1977) has shown that only pairs with separations $\geqslant 3000$ a.u. would likely dissolve within the lifetime of the galaxy; this is orders of magnitude wider than the separations typically catalogued for these nearby stars.

Thus, the VLI degenerates have not been found in spectroscopic and photometric surveying of faint single stars with large motions, or of bright stars with large motions. They haven't been found as binary companions, despite intense efforts by astrometrists to supersede the early discoveries of Van Biesbroeck. We must conclude that VLL white dwarfs are not present in anywhere near the numbers required by normalizing the $0.6 \mathrm{M}$ luminosity function with constant white dwarf birthrate to the Green (1977) empirical density of hot stars. Limits are brlefly discussed in the paper Liebert et al. (1979b). The luminosity function of white dwarfs probably peaks at $\mathrm{M}_{\text {bol }}=+15 \pm 1$.

\section{Implications of the Empirical Deficit of VLL Degenerates}

It is therefore appropriate to consider the three assumptions against which the empirical number of VLL stars was tested. More specifically, the deficiency might be accounted for if:

(1) the normalization of Green's (1977) density of hot degenerates is a serious overestimate;

(2) the theoretical cooling curve is badly in error, and the stars really do not spend much time at VLL, and/or

(3) the assumption of a constant white dwarf birthrate beyond $\sim 5 \times 10^{9}$ years ago--the cooling time at the beginning of the VLL interval--is an overestimate.

We can quickly dismiss (1) given the comprehensiveness of Green's survey, its consistency with earller determinations and a recent estimate of the hot white dwarf density by Chiu (1978).

The theoretical cooling function for a $0.6 \mathrm{M}_{\Theta}$ carbon/oxygen configuration was chosen to match the approximate mean mass of white dwarfs (which also have a narrow mass dispersion), as estimated by 
Koester, Schulz and Weidemann (1979). However, the Lamb and Van Horn (1975) $1 M_{\theta}$ calculation demonstrates that a more massive star should experience an earlier onset of Debye cooling, and spend somewhat less time in the VLL region. Shipman (1979) points out that the known sample of white dwarfs used for estimating mean parameters may be biased toward stars with preferentially large radii, and preferentially smaller masses. He suggests that the true mean mass may be nearer $0.75 \mathrm{M}_{\theta}$. However, the use of even the $1 \mathrm{M}_{\theta}$ Lamb and Van Horn curve would still predict substantial numbers of VLI stars. Furthermore, the analysis of Fontaine and Van Horn (1976) does not indicate that consideration of different envelope compositions is likely to change the predictions drastically.

Finally, there is the idea by Stevenson (1977) that heavy elements in the core might become insoluble at the time the main constituent--i.e. carbon--crystallizes. This could release enough gravitational energy to slow down the cooling process greatly, perhaps even suspending the star at the crystallization luminosity-say - 10-4L - -for the age of the galaxy. If this literally happens, there would be no VLL stars, but all of the stars predicted to be in the VLI interval and below would be found instead at the crystallization luminosity. Likewise, if interstellar accretion should become a dominant luminosity source at these low Iuminosities, the predictions are similar. A large factor excess near $10^{-4} \mathrm{~L}_{0}$ is certainly not precluded by the available data; the Stevenson hypothesis should be investigated quantitatively.

The third assumption of our "benchmark." that white dwarfs formed at a constant birthrate for the last $10^{10}$ years or so, is likely to be wrong. Its use permitted us to decouple the stellar birthrate considerations from the rest of the problem. However, generally favored star formation theories for the galactic disk predict that more stars and more white dwarfs would have been formed in the early history of the galaxy. If one folds in the approximate predictions of a Schmidt-type model, with a disk age of $-15 \times 10^{9}$ years (like that of the halo), the predicted numbers of VLL white dwarfs become substantially larger, the deficit becoming much worse! In this case, even the Stephenson idea doesn't appear capable of providing a theoretical rescue from the problem. Adensity of $z 100$ degenerates at the crystallization luminosity would be predicted within 10 pc.

A case can be made, however, that the "standard" galactic disk star formation picture is not correct. It is unlikely that the disk as a dynamical entity could originate billions of years after the halo was formed. However, unlike the white dwarfs discussed in 54, disks may grow markedly by steady accretion of diffuse external gas or of small irregular systems (c.f. Tinsley and Larson 1978). The infall of additional gas could enhance greatly the star formation rates at later times. We note that Demarque and McClure's (1977) analysis of old cluster and field giant H-R diagrams and Helfer's (1977) study of empirical stellar Iuminosity functions syggested at least that most star formation in the disk occurred $\leq 10^{10}$ years ago. If this were true, then the shortfall in VLL degenerates could be readily explained (c.f. D'Antona and Mazzitelli 1978). 
This work was supported by NSF grant AST 76-22273. I thank Drs. G. Rieke, M. Lebovsky and C. Dahn for the use of unpublished data.

\section{References}

Abt, H.A. and Levy, S.G. 1976, Ap.J.Suppl., 30, 273.

Alcock, C. 1979, paper presented at IAU Colloquium No. 53, July 1979. Alcock, C. an Illarionov, A. 1979, submitted to Ap.J.

Bלhm, K.H., Carson, T.R., Fontaine,G., and Van Horn, H.M. 1977, Ap.J., $217,521$.

Bessell, M.S. and Wickramasinghe, D.T. 1979, submitted to Publ.A.S.P.

Bessell, M.S., Wickramasinghe, D.T. and Cottrell, P. 1979, paper

presented at IAU Colloqium No. 53, July 1979.

Bues, I. 1970, Ast. Ap., 7, 91.

Bues, I. 1973, Ast. Ap., 28, 181.

Bues, I. 1979, paper presented at IAU Colloquium No. 53, July 1979.

Cottrell, P., Bessell, M.S. and Wickramasinghe, D.T. 1977, Ap.J. (Letters), 218, L133.

Dahn, C.C., Hintzen, P.M., Liebert, J., Stockman, H.S. and Spinrad, H. 1978, Ap.J., 219, 979.

D'Antona, F. and Mazzitelli, I. 1978, Ast. Ap., 66, 453.

D'Antona, F. and Mazzitelli, I. 1979a, Ast. Ap., 74, 161.

D'Antona, F. and Mazzitelli, I. 1979b, preprint.

Demarque, P. and McClure, R.D. 1977, in The Evolution of Galaxies and Stellar Populations, ed. B. M. Tinsley and R. B. Larson, Yale University, New Haven.

Eggen, 0.J. 1979a, Ap.J. Suppl., 39, 89.

Eggen, 0.J. 1979b, preprint.

Fontaine, G. and Van Horn, H.M. 1976, Ap.J. Suppl., 31, 467.

Green, R.F. 1977, PhD Thesis, California Institute of Technology, Pasadena, CA.

Gliese, W. 1969, A Catalogue of Nearby Stars, Veroff. Astron. RechenInst., Heidelberg, No. 22 .

Gliese, W. 1979, computer-printed updates to A Catalogue of Nearby Stars.

Greenstein, J.L. 1960, in Stars and Stellar Systems, Vol. 6, ed. J.I. Greenstein, Univ. of Chicago Press, Chicago.

Greenstein, J.L. 1970, in White Dwarfs, IAU Symposium No. 42, ed. W.J. Luyten, D. Reidel Publishing Co., Dordrecht.

Greenstein, J.I. 1976, Ap.J., 210, 524.

Greenstein, J.I. 1979, Ap.J., 227, 244.

Grenfell, T.C. 1974, Ast. Ap., 31, 303.

Helfer, H.L. 1977, preprint.

Hintzen, P.M., and Strittmatter, P.A. 1974, Ap.J.(Letters), 193, IIII. Hintzen, P.M. 1979, Publ.A.S.P., in press.

Humphreys, R.M., Iiebert, J., Romanishin,W. and Strittmatter, P.A. 1979, Publ.A.S.P., 91, 107.

King, I. 1977, Revista Mexicana de Astronomia y Astrofisica, 3, 167. Koester, D. 1976, Ast. Ap., 52, 415.

Koester, D., Schulz, H. and Weidemann, V. 1979, Ast. Ap., in press. Kovetz, A. and Shaviv, G. 1976, Ast. Ap., 52, 403. 
Lamb, D.Q. and Van Horn, H.M. 1975, Ap.J., 200, 306. Liebert, J. 1977, Ast. Ap., 56, 427.

Liebert, J. 1978, Ast. Ap., 70, 125.

Liebert, J., Dahn, C.C., Gresham, Mo, Hege, E.K., Moore, R.I., Romanishin, W. and Strittmatter, P.A. 1979a, Ap.J., 229, 196. Iiebert, J., Dahn, C.C., Gresham, M. and Strittmatter, P.A. 1979b, Ap.J., 233, in press.

Iuyten, W.J. 1976, The LHS Catalogue, University of Minnesota Press, Minneapolis.

Michaud, G. and Fontaine, G. 1979, Ap.J., 229, 694.

Mould, J. and Liebert, J. 1978, Ap.J.(Letters), 226, I29.

Shaviv, G. and Kovetz, A. 1976, Ast. Ap., 51, 383.

Shipman, H.I. 1977, Ap.J., 213, 138.

Shipman, H.L. 1979, Ap.J., 228, 240.

Sion, E.M. and Liebert, J. 1977, Ap.J., 213, 468.

Sion, E.M., Fragola, J.I. and O'Donnell, W.C. 1979, Publ. A.S.P., 91, in press,

Stevenson, D.J. 1977, Proc. Ast. Soc. Australia, 3, 167.

Strittmatter, P.A. and Wickramasinghe, D.T. 1971, Mon. Not. R.A.S., $152,47$.

Tinsley, B.M. and Larson, R.B. 1978, Ap.J., 221, 554.

Truran, J.W., Starrfield, S.G., Strittmatter, P.A., Wyatt, S.P., and Sparks, W.M. 1977, Ap.J., 211, 539.

Van Biesbroeck, G. 1944, Astron. J., 51, 61.

Van Biesbroeck, G. 1961, Astron. J., 66, 528.

Vauclair, G. and Reisse, C. 1977, Ast. Ap., 61, 415.

Vauclair, G. and Fontaine, G. 1979, Ap.J., in press.

Vauclair, G., Vauclair, S. and Greenstein, J.I. 1979, Ast. Ap., in press.

Wehrse, R. and Iiebert, J. 1979a, Ast. Ap., in press.

Wehrse, R. and Liebert, J. 1979b, Ast. Ap., in press.

Weidemann, V. 1960, Ap.J., 313, 638.

Weidemann, V. 1967, Z. I. Ap., 67, 286.

Wesemael, F. 1979, Ast. Ap., 72, 104.

Wickramasinghe, D.T. and Bessell, M.S. 1979, Mon.Not.R.A.S., 186, 399.

Wielen, R. 1974, in Highlights of Astronomy, 3, 395, ed. G. Contopoulos, D. Reidel Publishing Co., Dordrecht. 